\title{
Exotic nuclei with charm and bottom flavor
}

\author{
S. Yasui ${ }^{1, a}$ and K. Sudoh ${ }^{2}$ \\ 1 Institute of Particle and Nuclear Studies, High Energy Accelerator Research Organization (KEK), 1-1, Oho, Ibaraki, \\ 305-0801, Japan \\ 2 Nishogakusha University, 6-16, Sanbancho, Chiyoda, Tokyo, 102-8336, Japan
}

\begin{abstract}
We discuss the possibility of existence of exotic nuclei containing charm and bottom mesons. We study the interaction between $\bar{D}(B)$ mesons and nucleons from view of heavy quark symmetry, and derive the one pion exchange potentials. We apply these potentials to the two body system of $\bar{D}(B)$ meson and nucleon $N$, and find there are possible stable bound states with spin $J^{P}=1 / 2^{-}$and isospin $I=0$. We find that the tensor interaction mixing $\bar{D} N$ and $\bar{D}^{*} N\left(B N\right.$ and $\left.B^{*} N\right)$ plays an important role. We also qualitatively discuss the possible bound states of $\bar{D}(B)$ meson and two nucleons.
\end{abstract}

\section{Introduction}

The heavy hadrons with charm and bottom flavors are one of the most interesting fields of the quark and hadron physics. The heavy quark masses $m_{c}$ and $m_{b}$ of charm and bottom quarks introduce new energy scales in QCD, which are qualitatively different from the typical energy scale $\Lambda_{Q C D} \simeq 200 \mathrm{MeV}$ in light flavors. One of the typical features is the degeneracy of energy levels between pseudoscalar and vector mesons of heavy flavors in heavy quark mass limit. This is called as heavy quark symmetry [1]. For charm, in fact, we find the mass difference of $D$ and $D^{*}$ mesons is $m_{D^{*}}-m_{D} \simeq 140 \mathrm{MeV}$ [2]. For bottom, the mass difference is even smaller $m_{B^{*}}-m_{B} \simeq 45$ $\mathrm{MeV}$ [2]. Because their mass differences are almost equal or less than pion mass, these approximate degeneracies are regarded to represent examples of the heavy quark symmetry. Another interesting feature of heavy quark physics is that there are several effective theories directly based on QCD. For example, heavy quark effective theory (HQET) based on heavy quark symmetry has provided systematic understanding of heavy hadrons [1]. In degrees of freedom of mesons, the HQET are inverted to heavy meson effective theory $[1,3]$. The non-relativistic QCD (NRQCD) and potential non-relativistic QCD (pNRQCD) are also useful effective theories to study spectroscopy and generation processes of heavy quark systems [4]. The numerical simulation by lattice QCD is also a direct method to investigate the non-perturbative nature of QCD.

The hadrons with heavy flavors are now widely discussed not only in the normal hadrons composed with two and three quarks, but also in exotic hadrons recently discovered several accelerator facilities in the world $[5,6]$. The representative examples are $X(3872)$ [7], $Y(4260)$ [8], $Z^{ \pm}$(4430) [9], $Y_{b}$ [10] and so on. The field of study of the hadrons with the heavy flavors is also extended to the

\footnotetext{
a e-mail: yasuis@post.kek.jp
}

exotic nuclei containing charm and bottom flavors which may be accessible in high-energy hadron facilities such as J-PARC (Japan Proton Accelerator Research Complex) and GSI (Gesellschaft für Schwerionenforschung) [11]. In this paper, we study exotic nuclei with charm and bottom hadrons.

The study of exotic nuclei with charm and bottom flavors is important. One reason is that it is possible to apply the discussions starting from QCD by means of several effective theories derived from QCD, such as HQET, (p)NRQCD and so on. This is in contrast to the low energy effective theories for light flavors with chiral symmetry, in which there is no evident derivation from QCD. Another reason is that we can enlarge our knowledge of exotic nuclei, such as strangeness nuclei (hypernuclei and kanonic nuclei), which are now extensively studied in both theoretical and experimental sides [12,13]. The study of strangeness nuclei can be extended to the multi-flavor nuclei containing, not only strangeness, but also charm and bottom. These flavors play the role of impurity in the normal nuclei. One of the most interesting points in hypernuclei is that the mass scale of strange quarks may not be so much different from up and down quarks in nuclei, and hence it may open new types of nuclei with SU(3) flavor symmetry. On the other hand, the mass scale of charm and bottom quarks are qualitatively different from that of up and down quarks. Therefore, we expect that another dynamical scale characterized by heavy quark mass is introduced into the normal nuclei, there should be some qualitatively novel phenomena in heavy flavor nuclei. In any case, it would be important to understand the whole structure of exotic nuclei by investigating various kinds of flavor.

The study of exotic nuclei with heavy flavors can be traced back to the theoretical discussions of exotic nuclei with charm and bottom baryons, such as $\Lambda_{c}, \Sigma_{c}, \Lambda_{b}, \Sigma_{b}$ and so on [14]. The heavy flavor mesons in nuclei are also important issues. For closed charm mesons, $J / \psi$ in nuclei has 
been discussed by several authors in which effective theories of QCD [15] and lattice QCD studies [16] have been employed. For open charm mesons, $D$ meson in nuclei is an interesting subject [17-19]. In the present discussion, we study the exotic nuclei with $D(B)$ mesons as discussed in details in [19].

\section{Interactions from heavy quark symmetry}

One of the most basic question for study of the exotic nuclei with $D(B)$ mesons is to ask the interaction between $D$ $(B)$ mesons and nucleons. In many phenomenological studies, the $D$ mesons in nuclei have been discussed by means of the Weinberg-Tomozawa-type contact interaction with SU(4) flavor symmetry with $\pi, K$ and $D$ mesons [18]. This is a straightforward extension from successful SU(3) flavor symmetry with $\pi$ and $K$. However, because the mass scale of charm is much different form other light flavors, it is expected that the SU(4) symmetry would not be a good symmetry in nature. To grasp the reality, we shall see the mass spectroscopy of strange, charm and bottom mesons. In strangeness, the ground state is $K$ meson with mass about $500 \mathrm{MeV}$ and the next excited state is $K^{*}$ vector meson with mass about $892 \mathrm{MeV}$. Because of the large mass difference about $392 \mathrm{MeV}$ between $K$ and $K^{*}$ mesons, it is conventionally considered that the $K^{*}$ vector mesons are almost irrelevant degrees of freedom as far as we discuss the ground state. However, this is not the case for heavy flavors of charm and bottom. As indicated in the beginning of this paper, the mass difference between $D$ and $D^{*}$ mesons is about $140 \mathrm{MeV}$, and that of $B$ and $B^{*}$ mesons are even about $45 \mathrm{MeV}$. These mass difference can be regarded as small, because they are equal or less than pion mass. Therefore, it is important to consider not only $D(B)$ mesons, but also $D^{*}\left(B^{*}\right)$ mesons in the ground state.

The interaction between the $D(B)$ mesons and nucleons is qualitatively different from that of $K$ mesons and nucleons.To see this difference, let us start from strangeness. We remember the Weinberg-Tomozawa-type contact interaction for $K$ mesons and nucleons. It is well-known that the Weinberg-Tomozawa-type contact interaction can be regarded as exchange of $\omega$ and $\rho$ vector mesons. Here, one pion exchange process is absent in principle, because the coupling of $K K \pi$ is forbidden by parity conservation. In heavy flavor case, on the other hand, the couplings of $D D^{*} \pi$ and $D^{*} D^{*} \pi\left(B B^{*} \pi\right.$ and $\left.B^{*} B^{*} \pi\right)$ exist. Therefore, there is one pion exchange process in the interaction of $D(B)$ mesons and nucleons. This observation indicates that the $K N$ interaction is a short range force characterized with scale of mass of $\omega$ and $\rho$ mesons, while the $D N(B N)$ interactions are long range forces characterized with scale of mass of $\pi$ mesons.

The one pion exchange potential between $D(B)$ mesons and nucleons requires the information of $\pi$ meson and nucleon vertex, and $D(B)$ meson and nucleon vertex. The former is simply given by

$$
\mathcal{L}_{\pi N N}=-\frac{g_{\pi N N}}{m_{N}} \chi_{s^{\prime}}^{\dagger} \sigma \cdot \nabla \frac{\pi \cdot \tau}{2} \chi_{s}
$$

where $\chi_{s}$ is the nucleon field with spin $s, m_{N}$ is the nucleon mass, and $g_{\pi N N}^{2} / 4 \pi=13.5$ is the coupling constant. The latter is given with help of heavy quark symmetry,

$$
\mathcal{L}_{\pi H H}=g \operatorname{tr} \bar{H}_{a} H_{b} \gamma_{v} \gamma_{5} A_{b a}^{v},
$$

where the multiplet field $H$ of $P$ and $P^{*}$ is defined by

$$
H_{a}=\frac{1+\psi}{2}\left[P_{a \mu}^{*} \gamma^{\mu}-P_{a} \gamma_{5}\right]
$$

with the velocity $v$ of the mesons [3]. Here we define $P$ as $D$ or $B$, and $P^{*}$ as $D^{*}$ or $B^{*}$ for short notation. The conjugate field is $\bar{H}_{a}=\gamma_{0} H_{a}^{\dagger} \gamma_{0}$, and the index $a$ denotes up and down flavors. The axial current is given by $A_{\mu} \simeq \frac{i}{f_{\pi}} \partial_{\mu} \mathcal{M}$ with

$$
\mathcal{M}=\left(\begin{array}{cc}
\frac{\pi^{0}}{\sqrt{2}} & \pi^{+} \\
\pi^{-} & -\frac{\pi^{0}}{\sqrt{2}}
\end{array}\right),
$$

where $f_{\pi}=135 \mathrm{MeV}$ is the pion decay constant. The coupling constant $|g|=0.59$ for $\pi P P^{*}$ is determined from the observed decay width $\Gamma=96 \mathrm{keV}$ for $D^{*} \rightarrow D \pi$ [2]. The coupling of $\pi P^{*} P^{*}$, which is difficult to access from experiments, is automatically determined thanks to the heavy quark symmetry. Note that the coupling of $\pi P P$ does not exist due to the parity conservation. The coupling constant $g$ for $\pi B B^{*}$ would be different from one for $\pi D D^{*}$ because of $1 / m_{Q}$ corrections with the heavy quark mass $m_{Q}$ [20]. The recent lattice simulation in the heavy quark limit suggests the closed value adopted above [21]. This would allow us to use the common value for $D$ and $B$.

\section{Exotic bound states}

Now we move to discussion about the $P^{(*)} N$ bound states. In this analysis, we consider four states, whose quantum numbers are classified to $J^{P}=1 / 2^{-}$and $3 / 2^{-}$with isospin $I=0$ and 1 . The $1 / 2^{-}$states with $I=0$ and 1 are superpositions of three states, ${ }^{2} S_{1 / 2}$ for $P N$, and ${ }^{2} S_{1 / 2}$ and ${ }^{4} D_{1 / 2}$ for $P^{*} N$ with the standard notation ${ }^{2 S+1} L_{J}$. The $3 / 2^{-}$states with $I=0$ and 1 are superpositions of four states, ${ }^{2} D_{3 / 2}$ for $P N$, and ${ }^{4} S_{3 / 2},{ }^{4} D_{3 / 2}$, and ${ }^{2} D_{3 / 2}$ for $P^{*} N$. A similar analysis has been done in Ref. [22], in which they however do not take into account the mixing between different spin states, ${ }^{2} S_{1 / 2}$ in $P N$ and $P^{*} N$, and ${ }^{4} D_{1 / 2}$ in $P^{*} N$.

With these basis, we explicitly represent the potentials as [19]

$$
\begin{aligned}
V_{1 / 2^{-}} & =\frac{g g_{\pi N N}}{\sqrt{2} m_{N} f_{\pi}} \frac{1}{3} \\
& \times\left(\begin{array}{ccc}
0 & \sqrt{3} C_{\mu} & -\sqrt{6} T_{\mu} \\
\sqrt{3} C_{\mu} & -2 C_{m_{\pi}} & 0 \\
-\sqrt{6} T_{\mu} & 0 & C_{m_{\pi}}-2 T_{m_{\pi}}
\end{array}\right) \tau_{P} \cdot \tau_{N}, \\
V_{3 / 2^{-}} & =\frac{g g_{\pi N N}}{\sqrt{2} m_{N} f_{\pi}} \frac{1}{3} \\
& \times\left(\begin{array}{cccc}
0 & \sqrt{3} T_{\mu}-\sqrt{3} T_{\mu} & \sqrt{3} C_{\mu} \\
\sqrt{3} T_{\mu} & C_{m_{\pi}} & 2 T_{m_{\pi}} & T_{m_{\pi}} \\
-\sqrt{3} T_{\mu} & 2 T_{m_{\pi}} & C_{m_{\pi}} & -T_{m_{\pi}} \\
\sqrt{3} C_{\mu} & T_{m_{\pi}} & -T_{m_{\pi}} & -2 C_{m_{\pi}}
\end{array}\right) \tau_{P} \cdot \tau_{N},
\end{aligned}
$$


Table 1. The properties of the $\bar{D} N$ and $B N$ bound states for $J^{P}=$ $1 / 2^{-}$with $I=0$.

\begin{tabular}{lcc}
\hline & $\bar{D} N$ & $B N$ \\
\hline binding energy & $1.4 \mathrm{MeV}$ & $9.4 \mathrm{MeV}$ \\
relative radius & $3.8 \mathrm{fm}$ & $1.7 \mathrm{fm}$ \\
\hline
\end{tabular}

for $J^{P}=1 / 2^{-}$and $3 / 2^{-}$, respectively. $m_{\pi}$ is the pion mass and $\mu^{2}=m_{\pi}^{2}-\left(m_{P^{*}}-m_{P}\right)^{2}$ is the modified mass because of the different masses $m_{P^{*}}$ and $m_{P}$ for $P^{*}$ and $P . C_{m}$ and $T_{m}$ are defined as

$$
\begin{aligned}
& C_{m}=\int \frac{\mathrm{d}^{3} p}{(2 \pi)^{3}} \frac{1}{\mathbf{q}^{2}+m^{2}} e^{i \mathbf{q} \cdot \mathbf{r}} F(\mathbf{q} ; m), \\
& T_{m} S_{12}(\hat{r})=\int \frac{\mathrm{d}^{3} p}{(2 \pi)^{3}} \frac{-\mathbf{q}^{2}}{\mathbf{q}^{2}+m^{2}} S_{12}(\hat{q}) e^{i \mathbf{q} \cdot \mathbf{r}} F(\mathbf{q} ; m),
\end{aligned}
$$

with the tenor operator $S_{12}(\hat{x})=3\left(\sigma_{1} \cdot \hat{x}\right)\left(\sigma_{2} \cdot \hat{x}\right)-\sigma_{1}$. $\sigma_{2}$, and the form factor $F(\mathbf{q} ; m)=\left(\Lambda_{N}^{2}-m^{2}\right) /\left(\Lambda_{N}^{2}+\mathbf{q}^{2}\right) \times$ $\left(\Lambda_{P}^{2}-m^{2}\right) /\left(\Lambda_{P}^{2}+\mathbf{q}^{2}\right)$. Here we adopt $\Lambda_{N}=940 \mathrm{MeV}, \Lambda_{D}=$ $1266 \mathrm{MeV}$ and $\Lambda_{B}=1213 \mathrm{MeV}$ [19]. $\tau_{N}$ and $\tau_{P}$ are isospin operators for nucleon $N$ and meson $P$.

The kinetic terms are

$$
\begin{aligned}
K_{1 / 2^{-}}= & \operatorname{diag}\left(-\frac{1}{2 \widetilde{m}_{P}} \Delta_{0},-\frac{1}{2 \widetilde{m}_{P^{*}}} \Delta_{0}+\Delta m_{P P^{*}},\right. \\
& \left.-\frac{1}{2 \widetilde{m}_{P^{*}}} \Delta_{2}+\Delta m_{P P^{*}}\right), \\
K_{3 / 2^{-}}= & \operatorname{diag}\left(-\frac{1}{2 \widetilde{m}_{P}} \Delta_{2},-\frac{1}{2 \widetilde{m}_{P^{*}} \Delta_{0}+\Delta m_{P P^{*}},}\right. \\
& \left.-\frac{1}{2 \widetilde{m}_{P^{*}}} \Delta_{2}+\Delta m_{P P^{*}},-\frac{1}{2 \widetilde{m}_{P^{*}}} \Delta_{2}+\Delta m_{P P^{*}}\right),
\end{aligned}
$$

for $J^{P}=1 / 2^{-}$and $3 / 2^{-}$, respectively. Here we define $\Delta_{0}=$ $\partial^{2} / \partial r^{2}+(2 / r) \partial / \partial r$ and $\Delta_{2}=\Delta_{0}+6 / r^{2}, \widetilde{m}_{P^{(*)}}=m_{N} m_{P^{(*)}} /\left(m_{N^{+}}\right.$ $\left.m_{P^{(*)}}\right)$, and $\Delta m_{P P^{*}}=m_{P^{*}}-m_{P}$. The eigenvalue equation with the given hamiltonian, $H_{J^{P}}=K_{J^{P}}+V_{J^{P}}$ with $J^{P}=1 / 2^{-}$and $3 / 2^{-}$, is numerically solved by a variational method. The binding energy is realized as a difference from the threshold $m_{N}+m_{P}$.

We find the $\bar{D} N$ and $B N$ bound state solutions for $J^{P}=$ $1 / 2^{-}$with $I=0$ [19]. Their binding energies and the relative radii are $1.4 \mathrm{MeV}$ and $9.4 \mathrm{MeV}$, and $3.8 \mathrm{fm}$ and $1.7 \mathrm{fm}$, respectively, which are summarized in Table. 1. The wave functions are shown in Fig. 1, where the solid, dashed, and dotted curves represent $P N\left({ }^{2} S_{1 / 2}\right), P^{*} N\left({ }^{2} S_{1 / 2}\right)$, and $P^{*} N\left({ }^{4} D_{1 / 2}\right)$, respectively. The narrow (thick) curves show the $\bar{D} N(B N)$ states. We indicate that the $P^{*} N\left({ }^{4} D_{1 / 2}\right)$ component is crucially important, though it is smaller than the $P N\left({ }^{2} S_{1 / 2}\right)$ as shown in Fig. 1. The binding energy is induced mainly by the tensor coupling between $P N\left({ }^{2} S_{1 / 2}\right)$ and $P^{*} N\left({ }^{4} D_{1 / 2}\right)$, which are in off-diagonal terms in $V_{1 / 2^{-}}$ in Eq. (5). Indeed, without this tensor coupling, we cannot obtain any bound state. Thus, the $P N-P^{*} N$ mixing plays an essential role in these systems.

We see that the $B N$ state is more deeply bound and compact than the $\bar{D} N$ state. One reason is that the smaller

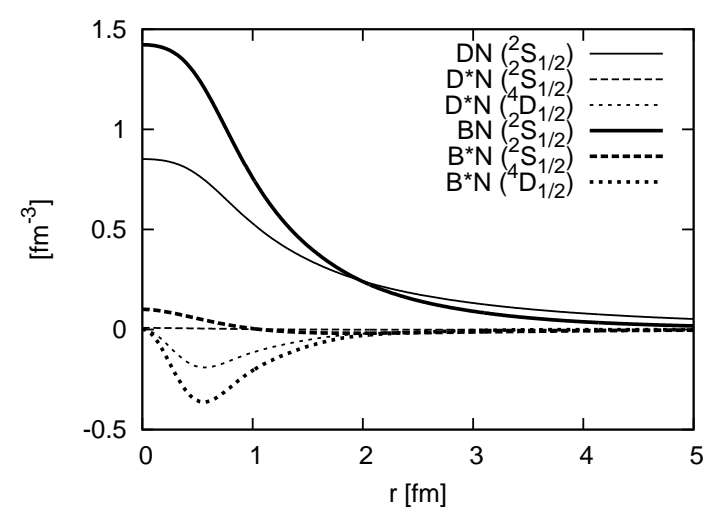

Fig. 1. The wave functions of the $\bar{D} N$ and $B N$ bound states for $J^{P}=1 / 2^{-}$with $I=0$.

mass splitting between $B$ and $B^{*}$ strengthens the $B N-B^{*} N$ mixing. Another reason is that the kinetic energy of $B$ mesons is suppressed simply due to heavy mass. In the heavy quark limit, the complete mass degeneracy of $P$ and $P^{*}$ induces the ideal $P N-P^{*} N$ mixing, which would give the maximal binding energy.

It is worth to emphasize that we can not find $\bar{D} N$ and $B N$ bound states for other channels, $J^{P}=1 / 2^{-}$with $I=1$, and $J^{P}=3 / 2^{-}$with $I=0$ or 1 . As a result, we conclude that $J^{P}=1 / 2^{-}$with $I=0$ are the most promising channel for detecting stable $\bar{D} N$ and $B N$ bound states.

In experiments, the $\bar{D} N$ and $B N$ bound states would be searched in $e^{+} e^{-}$collisions, or anti-proton beam with deuteron targets $[23,24]$. We note there is no open decay channel below the threshold, into which the $\bar{D} N$ and $B N$ bound states decay by strong interaction. Therefore, they are stable objects and experimentally well accessible despite of their small binding energies. The $\bar{D} N$ and $B N$ wave functions have the following components,

$$
\begin{aligned}
& |\bar{D} N\rangle=\left(\left|D^{-} p\right\rangle-\left|\bar{D}^{0} n\right\rangle\right)+\left(\left|D^{*-} p\right\rangle-\left|\bar{D}^{* 0} n\right\rangle\right), \\
& |B N\rangle=\left(\left|B^{0} p\right\rangle-\left|B^{+} n\right\rangle\right)+\left(\left|B^{* 0} p\right\rangle-\left|B^{*+} n\right\rangle\right),
\end{aligned}
$$

with neglecting the weight of each component. We find that the weak decay processes, $D^{-} p \rightarrow K^{+} \pi^{-} \pi^{-}+p$ and $B^{0} p \rightarrow$ $D^{-} \pi^{+}+p$, in the first component would be available for reconstruction of the invariant mass.

The $\bar{D} N$ and $B N$ bound states contain five quarks as ingredient. Therefore they can be compared with the $K N$ states as candidate of pentaquarks [25]. It has been theoretically studied that the $K$ meson is not bound with a nucleon by the Weinberg-Tomozawa interaction with chiral symmetry [26]. In contrast, our analysis shows the $\bar{D}$ and $B$ mesons are bound with a nucleon by one pion exchange potential with respecting heavy quark symmetry. Therefore, the $\bar{D} N$ and $B N$ bound states may open another way to search for pentaquark with heavy flavors [27-29]. 


\section{Toward exotic nuclei}

The work on $\bar{D} N$ bound states is extended to exotic nuclei containing $\bar{D}$ mesons. One can expect that the binding energy of the $\bar{D}$ meson in such exotic nuclei becomes larger as baryon number increases. Such exotic nuclei will be investigated in J-PARC and GSI by using anti-proton beams with targets of nuclei $[30,31]$. Here we discuss briefly the $\bar{D} N N$ states with baryon number two. The possible states for $I=1 / 2$ are classified to $\left|\bar{D}(N N)_{0,1}\right\rangle+\left|\bar{D}^{*}(N N)_{1,0}\right\rangle$ with $J^{P}=0^{-}$and $\left|\bar{D}(N N)_{1,0}\right\rangle+\left|\bar{D}^{*}(N N)_{1,0}\right\rangle+\left|\bar{D}^{*}(N N)_{0,1}\right\rangle$ with $J^{P}=1^{-}$. For $I=3 / 2$, they are $\left|\bar{D}(N N)_{0,1}\right\rangle$ with $J^{P}=0^{-}$and $\left|\bar{D}^{*}(N N)_{0,1}\right\rangle$ with $J^{P}=1^{-}$, where subscripts denote the spin and isospin of nucleon pairs. Only the former two states for $I=1 / 2$ contain the attractive $\bar{D} N$ pairs for $J^{P}=1 / 2^{-}$with $I=0$. Therefore, one can expect that the $\bar{D} N N$ states, as well as the $B N N$ states, for $J^{P}=0^{-}$and/or $1^{-}$with $I=1 / 2$ would be stable.

It is valuable to compare the $\bar{D} N N$ and $B N N$ states with the $K^{-} p p$ states $\left(\bar{K}(N N)_{0,1}\right.$ in our notation) [32]. The $K^{-} p p$ state is considered to have large binding energy 20 $70 \mathrm{MeV}$. However, the $K^{-} p p$ state decays to $\pi Y N$ by a strong interaction with the decay width $40-70 \mathrm{MeV}$. On the other hand, the $\bar{D} N N$ and $B N N$ states are stable in the strong decay as discussed above. Therefore, the $\bar{D} N N$ and $B N N$ states may provide more precise information about exotic nuclei. More quantitative analyses including fewbody calculation will be presented in Ref. [33].

The charmed and bottom nuclei like $\bar{D} N N$ and $B N N$ states are comparable with the hypothetical existence of the kaonic nuclei, in which $K$ mesons are bound in nuclei [34]. The existence of such nuclei may realize if there is a sufficient number of nucleons around $K$ meson, though $K N$ interaction itself seems not attractive to form $K N$ molecule [26]. We stress again that in our analysis the $\bar{D}$ and $B$ mesons have sufficiently large attraction to form the stable charmed and bottom nuclei beginning with baryon number one.

Finally we mention the possibility that the study of $\bar{D}$ and $B$ nuclei may extend our understanding of exotic nuclei with multi-flavors. It is known that there is a mixing of $\Lambda N-\Sigma N$ in hypernuclei [13]. This mixing enhances the binding energy of $\Lambda$ in hypernuclei. In fact, without this mixing, we cannot explain the observed binding energy of $\Lambda$ in several experiments. The importance of $\Lambda N-\Sigma N$ mixing reminds us the role of $\bar{D} N-\bar{D}^{*} N\left(B N-B^{*} N\right)$ mixing in the present discussion. In our case, too, the mixing plays an important role to supply effective attraction to form bound states of $\bar{D} N$ and $B N$. Although we are not yet in a position to discuss more details, this similarity seems to suggest a dual relationship of hypernuclei containing hyperon, and charm and bottom exotic nuclei containing $\bar{D}$ and $B$ mesons.

\section{Summary}

In summary, bound states of nucleon and $\bar{D}$ and $B$ meson are discussed with respecting the heavy quark symmetry.
It is found $\bar{D} N$ and $B N$ bound states with binding energies $1.4 \mathrm{MeV}$ and $9.4 \mathrm{MeV}$, respectively, for $J^{P}=1 / 2^{-}$ with $I=0$, and no bound states in other channels. These states are stable in the strong decay, and can be observed in the weak decay processes $\bar{D} N \rightarrow K^{+} \pi^{-} \pi^{-}+p$, and $B N \rightarrow D^{-} \pi^{+}+p$. The existence of $\bar{D} N$ and $B N$ bound states would provide an opportunity to probe new exotic states near the thresholds, and open a new way to investigate for exotic nuclei with variety of multi-flavor explored at future hadron facilities such as J-PARC and GSI.

The authors thank A. Dote for fruitful discussions.

\section{References}

1. For example, A. V. Manohar and M. B. Wise, Camb. Monogr. Part. Phys. Nucl. Phys. Cosmol. 10 (2000) 1.

2. C. Amsler et al. [Particle Data Group], Phys. Lett. B 667 (2008) 1.

3. N. Isgur and M. B. Wise, Phys. Rev. Lett. 66 (1991) 1130; G. Burdman and J. F. Donoghue, Phys. Lett. B 280 (1992) 287; M. B. Wise, Phys. Rev. D 45 (1992) R2188; T. M. Yan, H. Y. Cheng, C. Y. Cheung, G. L. Lin, Y. C. Lin and H. L. Yu, Phys. Rev. D 46 (1992) 1148 [Erratum-ibid. D 55 (1997) 5851]; M. A. Nowak, M. Rho and I. Zahed, Phys. Rev. D 48 (1993) 4370; R. Casalbuoni, A. Deandrea, N. Di Bartolomeo, R. Gatto, F. Feruglio and G. Nardulli, Phys. Rept. 281 (1997) 145; W. A. Bardeen, E. J. Eichten and C. T. Hill, Phys. Rev. D 68 (2003) 054024; T. Matsuki, T. Morii and K. Sudoh, Prog. Theor. Phys. 117 (2007) 1077

4. N. Brambilla, A. Pineda, J. Soto and A. Vairo, Rev. Mod. Phys. 77 (2005) 1423.

5. E. S. Swanson, Phys. Rept. 429 (2006) 243.

6. M. B. Voloshin, Prog. Part. Nucl. Phys. 61 (2008) 455.

7. S. K. Choi et al. [Belle Collaboration], Phys. Rev. Lett. 91 (2003) 262001; B. Aubert et al. [BABAR Collaboration], Phys. Rev. D 71 (2005) 071103.

8. C. Z. Yuan et al. [Belle Collaboration], Phys. Rev. Lett. 99 (2007) 182004.

9. S. K. Choi et al. [BELLE Collaboration], Phys. Rev. Lett. 100 (2008) 142001.

10. K. F. Chen et al. [Belle Collaboration], Phys. Rev. Lett. 100 (2008) 112001.

11. M. F. Lutz, B. Pire, O. Scholten and R. Timmermans [The PANDA Collaboration], arXiv:0903.3905 [hepex].

12. Y. Akaishi and T. Yamazaki, Phys. Rev. C 65 (2002) 044005.

13. A. Gal, arXiv:0904.4009 [nucl-th], and references cited therein.

14. C. B. Dover and S. H. Kahana, Phys. Rev. Lett. 39 (1977) 1506; S. Iwao, Lett. Nuovo Cim. 19, 647 (1977); R. Gatto and F. Paccanoni, Nuovo Cim. A 46 (1978) 313; H. Bando and M. Bando, Phys. Lett. B 109 (1982) 164; G. Bhamathi, Phys. Rev. C 24 (1981) 1816. 
15. S. J. Brodsky et al., Phys. Rev. Lett. 64 (1990) 1011;

A. Hayashigaki, Prog. Theor. Phys. 101 (1999) 923;

V. B. Belyaev et al., Nucl. Phys. A 780 (2006) 100.

16. K. Yokokawa, S. Sasaki, T. Hatsuda and A. Hayashigaki, Phys. Rev. D 74 (2006) 034504.

17. K. Tsushima, D. H. Lu, A. W. Thomas, K. Saito and R. H. Landau, Phys. Rev. C 59 (1999) 2824; A. Mishra, E. L. Bratkovskaya, J. Schaffner-Bielich, S. Schramm and H. Stocker, Phys. Rev. C 69 (2004) 015202; T. Mizutani and A. Ramos, Phys. Rev. C 74 (2006) 065201;

18. M. F. M. Lutz and E. E. Kolomeitsev, Nucl. Phys. A 730 (2004) 110; J. Hofmann and M. F. M. Lutz, Nucl. Phys. A 763 (2005) 90; D. Gamermann, E. Oset, D. Strottman and M. J. Vicente Vacas, Phys. Rev. D 76 (2007) 074016; J. Haidenbauer, G. Krein, U. G. Meissner and A. Sibirtsev, Eur. Phys. J. A 33 (2007) 107.

19. S. Yasui and K. Sudoh, Phys. Rev. D 80 (2009) 034008.

20. H. Y. Cheng, C. Y. Cheung, G. L. Lin, Y. C. Lin, T. M. Yan and H. L. Yu, Phys. Rev. D 49 (1994) 2490; C. G. Boyd and B. Grinstein, Nucl. Phys. B 442 (1995) 205.

21. H. Ohki, H. Matsufuru and T. Onogi, Phys. Rev. D 77 (2008) 094509.

22. T. D. Cohen, P. M. Hohler and R. F. Lebed, Phys. Rev. D 72 (2005) 074010.

23. A. Sibirtsev, Nucl. Phys. A 680 (2000) 274.

24. J. Haidenbauer, G. Krein, U. G. Meissner and A. Sibirtsev, Eur. Phys. J. A 37 (2008) 55.

25. T. Nakano et al. [LEPS Collaboration], Phys. Rev. C 79 (2009) 025210.

26. T. Hyodo, D. Jido and A. Hosaka, Phys. Rev. Lett. 97 (2006) 192002, and references therein.

27. Y. Oh, B. Y. Park and D. P. Min, Phys. Rev. D 50 (1994) 3350; Y. s. Oh and B. Y. Park, Z. Phys. A 359 (1997) 83.

28. S. H. Lee, S. Yasui, W. Liu and C. M. Ko, Eur. Phys. J. C 54 (2008) 259.

29. S. H. Lee and S. Yasui, Eur. Phys. J. C 64 (2009) 283.

30. Y. S. Golubeva, W. Cassing and L. A. Kondratyuk, Eur. Phys. J. A 14 (2002) 255.

31. A. Sibirtsev, K. Tsushima and A. W. Thomas, Eur. Phys. J. A 6 (1999) 351.

32. A. Dote, T. Hyodo and W. Weise, Phys. Rev. C 79 (2009) 014003.

33. K. Sudoh and S. Yasui, in preparation.

34. A.S. Goldhaber, Proceedings of the Second LAMPF II Workshop, edited by H.A. Thiessen, T.S. Bhatia, R.D. Carlini, and N. Hintz, LA-9572-C, Vol. I (1982) 171. 